\title{
First-principles modeling of geomagnetically induced electromagnetic fields and currents from upstream solar wind to the surface of the Earth
}

\author{
A. Pulkkinen ${ }^{1,2}$, M. Hesse ${ }^{2}$, M. Kuznetsova ${ }^{2}$, and L. Rastätter ${ }^{2,3}$ \\ ${ }^{1}$ Goddard Earth Sciences and Technology Center, University of Maryland, Baltimore, MD, USA \\ ${ }^{2}$ NASA/GSFC, Greenbelt, Code 674, MD 20771, USA \\ ${ }^{3}$ Catholic University, Washington D.C., USA
}

Received: 20 November 2006 - Revised: 5 April 2007 - Accepted: 13 April 2007 - Published: 8 May 2007

\begin{abstract}
Our capability to model the near-space physical phenomena has gradually reached a level enabling modulebased first-principles modeling of geomagnetically induced electromagnetic fields and currents from upstream solar wind to the surface of the Earth. As geomagnetically induced currents (GIC) pose a real threat to the normal operation of long conductor systems on the ground, such as high-voltage power transmission systems, it is quite obvious that success in accurate predictive modeling of the phenomenon would open entirely new windows for operational space weather products.

Here we introduce a process for obtaining geomagnetically induced electromagnetic fields and currents from the output of global magnetospheric MHD codes. We also present metrics that take into account both the complex nature of the signal and possible forecasting applications of the modeling process. The modeling process and the metrics are presented with the help of an actual example space weather event of 24-29 October 2003. Analysis of the event demonstrates that, despite some significant shortcomings, some central features of the overall ionospheric current fluctuations associated with GIC can be captured by the modeling process. More specifically, the basic spatiotemporal morphology of the modeled and "measured" GIC is quite similar. Furthermore, the presented user-relevant utility metrics demonstrate that MHD-based modeling can outperform simple GIC persistence models.
\end{abstract}

Keywords. Ionosphere (Auroral ionosphere; Modeling and forecasting) - Space plasma physics (Numerical simulation studies)

Correspondence to: A. Pulkkinen

(antti.pulkkinen@gsfc.nasa.gov)

\section{Introduction}

Over the past few years there has been great progress in establishing extensive space weather frameworks having an ambitious goal of module-based modeling of the space weather phenomenon from the Solar surface to the planetary ionospheres (see Tóth et al., 2005, and references therein). Obviously, self-consistent modeling of physical phenomena in near-Earth space provides an unprecedented capability to gain new understanding about the Solar surface, corona, heliosphere, magnetosphere and ionosphere systems behavior as a whole. However, although much of space weather is covered within the Solar surface and Earth's ionosphere, the space weather phenomena do not end there; the processes extend down to the surface and below the surface of the Earth in terms of geomagnetic induction driven by variations in the near-space current systems.

From the applications viewpoint, extending the space weather frameworks to include the geomagnetic induction component provides new tools for mitigating geomagnetically induced currents (GIC) flowing in long conductor systems on the ground (e.g., Boteler et al., 1998; Molinski, 2002; Pirjola et al., 2004). Based on the sole impact of the famous March 1989 storm on the North American power transmission system (e.g., Czech et al., 1992; Bolduc, 2002), it is safe to say that GIC is one of the most important space weather hazards and that need for science-based mitigation capabilities is real. However, the present mitigation capabilities are limited mostly to statistical estimates based on historical data and to nowcasting of GIC levels (e.g., Pulkkinen et al., 2001b; Viljanen et al., 2006b; Boteler et al., 2006). It follows that success in accurate modeling enabling forecasting discussed in this work would open a new avenue for sciencebased GIC mitigation with true potential for commercial applications. Obviously, such applications would give an

Published by Copernicus GmbH on behalf of the European Geosciences Union. 
appealing additional societal justification for the work put into the large number of space physical models involved in the modeling chain.

Work presented here is by no means the first attempt to use information from the upstream solar wind to estimate the level of GIC fluctuations. Relatively successful empirical efforts have been made to reproduce some features of GIC, or proxies of GIC (Weigel et al., 2003; Wintoft, 2005). However, although similar activities are underway at the Darthmouth College ${ }^{1}$ and some properties of the time derivative of the ground magnetic field given by global magnetohydrodynamic (MHD) modeling have been analyzed by Raeder et al. (2001b), the work at hand is the first reported attempt to carry out GIC modeling by using first-principles models. By first-principles modeling we refer here to the process of solving the evolution equations of the system, or coupled systems as is done here, derived using elementary physical principles. This is in contrast to empirical modeling procedures where observations are used to derive the equations. Although empirical models have their important role in acquiring understanding about the behavior of the system, a first-principles approach is the natural ultimate goal for the modeling of any physical system. Thus, although the well-known limitations of, for example, MHD modeling (these limitations will be discussed more in detail below) lower some of the expectations for the accuracy of the final output of our interest, it is worthwhile to start moving toward this goal.

The work presented here comprises Phase 1 of the project dedicated to test the present capabilities of first-principles modeling of space weather from the ground and especially from the GIC viewpoint. More specifically, in the work at hand we will present the basic modeling process for obtaining the quantities of our interest from the output of the MHD codes. We will also explore metrics that take into account both the complex nature of the signal and possible forecasting applications of the modeling process; the selected metrics will be one of the backbones of the future work. Although the modeling process and the metrics are introduced with the help of an actual example space weather event of 2429 October 2003, rigorous testing utilizing different model setups and framework components will be carried out later in Phase 2 of the project.

The structure of the paper is as follows. First, in Sect. 2 we will go through the various steps of the modeling process leading to induced electromagnetic fields on the ground. Once the induced electric field is known, GIC in individual technological systems can be easily computed. In Sect. 3, we will model the space weather event of 24-29 October 2003. The output, geomagnetically induced electromagnetic fields and currents, are then compared to the measured quantities and the performance of the modeling chain is evaluated qualitatively. In Sect. 4, we explore the metrics appropriate for

\footnotetext{
${ }^{1}$ http://engineering.dartmouth.edu/ $\sim$ simon/research/GIC/index. html
}

quantifying the model performance especially from the GIC viewpoint. The model output of the example event is then evaluated using the selected metrics. Finally, in Sect. 5 we briefly summarize and discuss the findings of the study.

\section{Modeling process}

The core of the modeling process realized here is composed of the three-dimensional (3-D) global magnetospheric MHD code BATS-R-US (Powell et al., 1999) coupled to a twodimensional electrostatic ionospheric inner boundary model (Ridley et al., 2004). The code was driven using convection delayed L1 solar wind plasma and magnetic field observations for a period of 24 October 2006 11:28 UT-29 October 2006 06:28 UT (see Fig. 1). Without trying to make any more definite estimation for the errors here, it is noted that inaccuracies in the solar wind propagation to the model boundary will inevitably lead to inaccuracies in the timing of the modeled field fluctuations (see, e.g., Bargatze et al., 2005, and references therein). This issue will be studied more in detail in the follow-up of this initial study. The run was carried out utilizing the facilities at the Community Coordinated Modeling Center (CCMC) operating at NASA Goddard Space Flight Center and the state of the system was recorded every $4 \mathrm{~min}$, which dictated the temporal resolution of further modeling. Consequently, all data presented in this paper are averaged to a 4-min time resolution. On the ionospheric side, auroral conductance model was used in the ionospheric potential solver. We used a relatively sparse magnetospheric grid, the minimum cell width being $0.5 R_{E}$ (Earth radii), which resulted in about 300000 global MHD model cells. The objective in using such a poor resolution was to be able to carry out the run in a realistic operational setup; the same setup is used currently at CCMC for real-time runs of the BATSR-US. These runs are made with three parallel nodes each of which are powered by 2 AMD Opteron $2.2 \mathrm{GHz}$ model 248 processors. We note that massively parallel BATS-R-US code scales very well with the number of processors; supercomputers with $\sim 100$ processors enable real-time runs with 1.3 million model cells (Tóth et al., 2005).

The following process was used to compute the induced electromagnetic fields at the surface of the Earth (see Fig. 2): 1.) Ionospheric currents produced by the BATS-R-US were transformed from geomagnetic coordinates to geographic coordinates (GEO). 2.) A point $\left(\theta_{0}, \lambda_{0}\right)$ in GEO was selected to which fields are computed. 3.) Horizontal ionospheric currents within a radius of $1000 \mathrm{~km}$ about $\left(\theta_{0}, \lambda_{0}\right)$ were determined for each time step. Note that as in the auroral region ionospheric currents close to $\left(\theta_{0}, \lambda_{0}\right)$ typically dominate the external ground magnetic field fluctuations at $\left(\theta_{0}, \lambda_{0}\right)$, computationally very expensive inclusion of the entire ionosphere is not necessary. 4.) The time series of the horizontal ionospheric currents within radius of $1000 \mathrm{~km}$ about $\left(\theta_{0}, \lambda_{0}\right)$ were transformed to Cartesian coordinates using the 

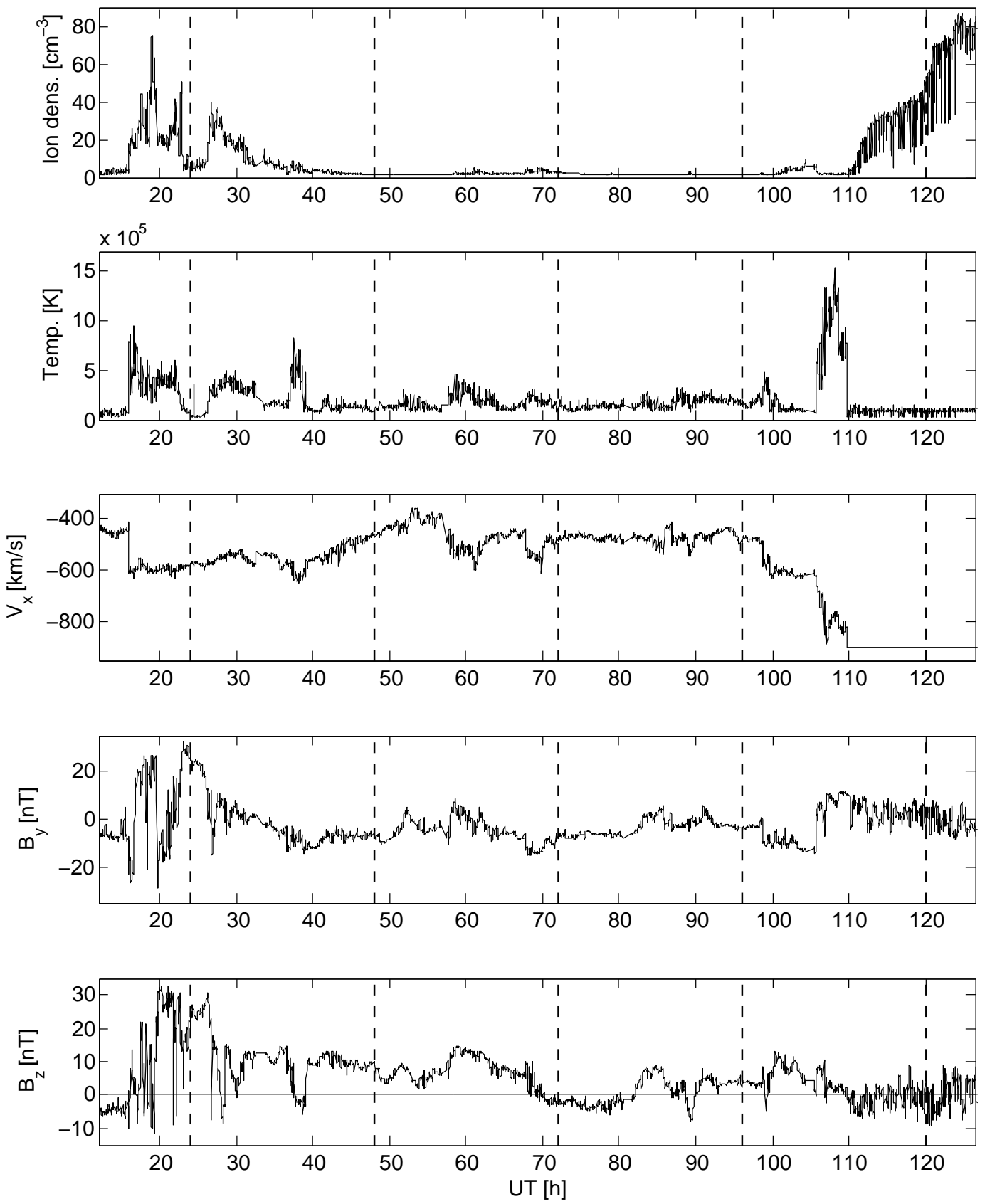

Fig. 1. The solar wind driver of the BATS-R-US run. The time is UT hours from the beginning of 24 October 2003 . The vertical dashed lines indicate the beginning of each new UT day and the solid straight line in the bottom panel indicates the zero-level.

stereographic projection and the currents were interpolated to a uniform rectangular Cartesian grid. 5.) Additional currents carrying out the current essentially from and to infinity were added to the edges of the Cartesian grid. This step is necessary to avoid the strong artificial field-aligned currents at the edges of the grid. The additional currents eliminate the fieldaligned currents at the edges completely. 6.) The Complex Image Method (CIM) (Boteler and Pirjola, 1998; Pirjola and Viljanen, 1998) was used to compute the ground response to the ionospheric driving with given one-dimensional (1-
D) ground conductivity structure. In using CIM we assume that field-aligned currents are exactly vertical and we decompose the ionospheric currents into U-shaped current elements; field-aligned currents at the two points of the Cartesian grid and a horizontal current filament with equal current amplitude in between these points. CIM computations are carried out in the spectral domain, transformations between the temporal and the spectral domains were carried out using Fast Fourier Transformation. 

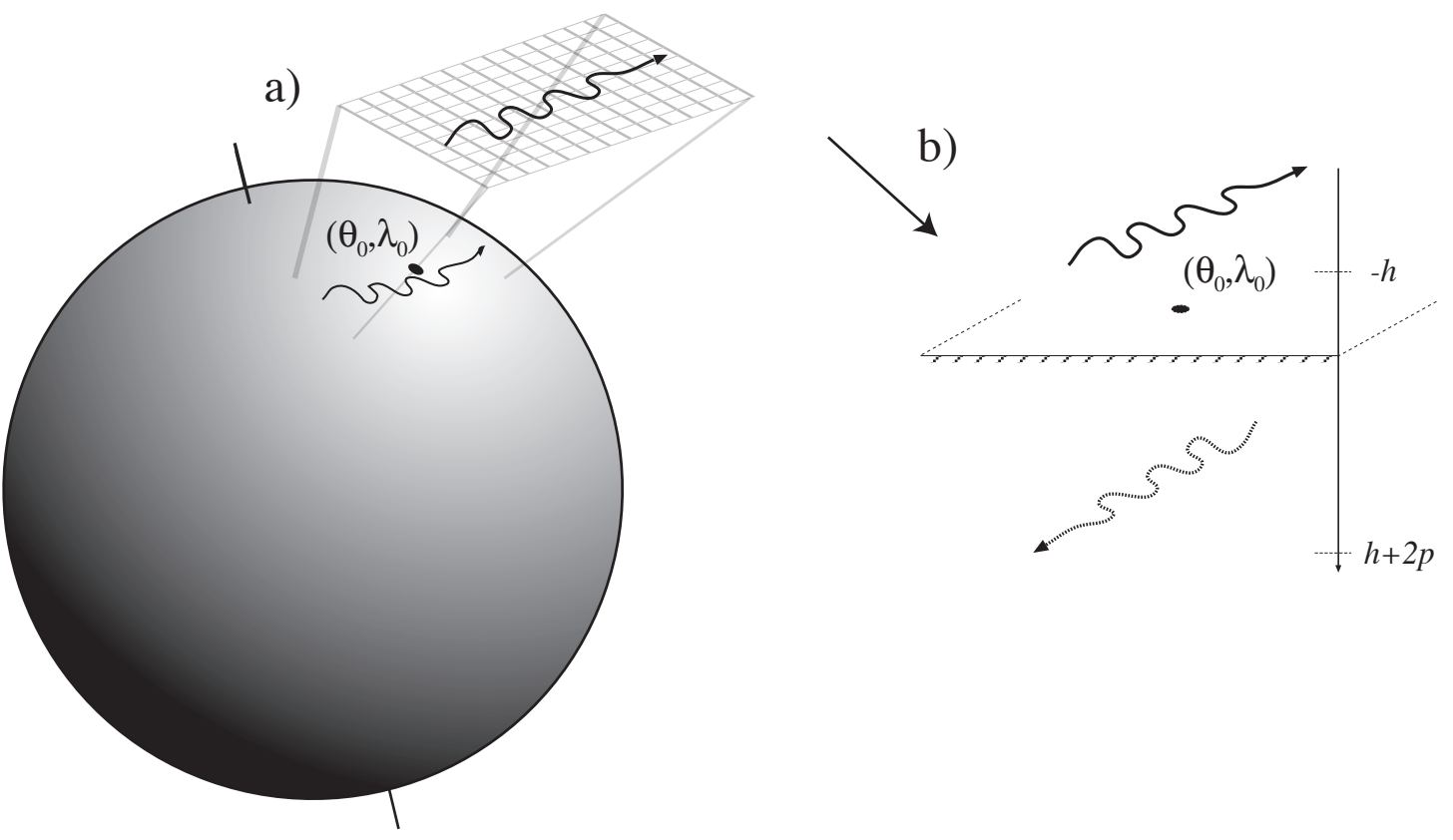

Fig. 2. Schematic view to the key steps for computing geomagnetically induced electromagnetic fields from the ionospheric output of the global MHD codes. (a) The time series of the horizontal ionospheric currents within a radius of $1000 \mathrm{~km}$ about $\left(\theta_{0}, \lambda_{0}\right)$ are transformed to Cartesian coordinates using the stereographic projection and the currents are interpolated to a uniform rectangular grid. (b) image of the original current system at the height $h$ is placed to the depth $h+2 p$. $p$ is the complex depth determined using the 1-D conductivity structure of the Earth (Pirjola and Viljanen, 1998). The total field at $\left(\theta_{0}, \lambda_{0}\right)$ is a superimposition of the fields produced by the original current at height $h$ and the image current at depth $h+2 p$.

The process used to compute the induced electromagnetic fields contains simplifications that require some discussion. First, only the local (within radius of $1000 \mathrm{~km}$ ) ionospheric current variations are used to drive the geomagnetic induction. Although this is a generally valid approach for the auroral ionosphere which is relatively close to the surface of the Earth, the same simplification cannot be used if more distant currents, for example the ring current, are used. However, as was mentioned above, inclusion of currents from large areas is computationally expensive and thus minimization of the space over which the integration is made is preferable. Also the usage of the Cartesian geometry requires spatial locality; a part of the surface of the sphere having a radius of $R_{E}$ cannot really be described as a plane for distances greater than about $1000 \mathrm{~km}$. Additionally, we need to assume that the field-aliged currents are exactly vertical (Pirjola and Viljanen, 1998). This is a generally valid assumption in auroral ionospheric studies (e.g., Untiedt and Baumjohann, 1993) but the same is not true for lower latitudes where the inclination of the currents is far from vertical. Finally, at present CIM is formulated to be applicable only to studies where the ground conductivity is 1-D, i.e. there are no horizontal conductivity gradients. Although 1-D approximation has been shown to be valid in numerous space weather-related geomagnetic induction studies (see e.g., Viljanen et al., 2004; Pulkkinen and Engels, 2005), there are important special cases where horizontal gradients cannot be neglected. Such cases include, for example, boundaries of continents and deep oceans where the so-called "coast effect" plays an important role in modifying the induced electromagnetic fields (e.g., Beamish et al., 2002; Olsen and Kuvshinov, 2004). Due to the relatively shallow sea-shores, the close proximity of the sea to some of the ground magnetometer stations used in this study does not severely distort the validity of the 1-D approximation. Note that 1-D model can be varied as a function of $\left(\theta_{0}, \lambda_{0}\right)$, which enables approximate treatment of lateral variations in the ground response.

What follows from the considerations above is that the procedure used to compute the induced electromagnetic fields is tailored for auroral ionospheric studies; a more general approach is necessary for other types of ionospheric/magnetospheric input. However, in the context of space weather, as the most intense induced fields and thus also the greatest technological hazards are experienced in the auroral regions, the simplified approach is justified. Moreover, the 1-D approach that neglects horizontal gradients of the ground conductivity is numerically much lighter than, for example, full 3-D geomagnetic induction modeling (see e.g., Avdeev et al., 2002). This is obviously beneficial if possible operational activities are kept in mind. Anyway, with the introduced modeling process the numerical bottleneck is the magnetospheric MHD, not the computations associated with the geomagnetic induction. 


\section{24-29 October 2003 event and the qualitative valida- tion of the model performance}

The geomagnetically active period of 24-29 October 2003 was a prelude for the extremely intense Halloween storms of 29-31 October 2003 (for a GIC view to the storm, see Pulkkinen et al., 2005). However, despite the storm strength of the period of our interest was significantly smaller than that of the Halloween storms, there was notable auroral ionospheric activity throughout 24-29 October 2003 as will be seen below. It follows, that the period is of interest also from the space weather viewpoint. In addition, it is quite clear that extreme storms require extreme model performance and thus it is worthwhile to start the modeling and the subsequent analysis with more moderate challenges.

The solar wind driver of the MHD code is shown in Fig. 1. It is seen that especially in terms of the $B_{z}$ component of the interplanetary magnetic field (IMF), the driving was only moderate. However, there were several negative "sweeps" of $B_{z}$ each causing enhanced ionospheric activity. This is true especially at the end of the event where the increasing solar wind speed and highly fluctuating but on average negative $B_{z}$, the beginning of the Halloween storm sequence, causes already quite notable ionospheric activity. It should be noted that L1 solar wind plasma observations, especially proton density recordings contain significant inaccuracies for the Halloween storm event (Skoug et al., 2004; Dmitriev et al., 2005). These problems, however, affect our analysis only at the very end of the period (starting from about 11:00 UT in Fig. 1) where the solar velocity and density are uncertain.

The points $\left(\theta_{0}, \lambda_{0}\right)$ on the ground at which we compute the modeled fields are shown in Fig. 3. These are the locations from which we obtained measured ground magnetic field data to be used in analyzing the model performance. Figure 3 also shows a snapshot of the ionospheric output of the BATSR-US. From this one of the shortcomings of the model run becomes apparent: the inner magnetospheric boundary of $3 R_{E}$ mapped to the ionosphere along the magnetic field lines does not extend very low in magnetic latitude. This should be considered as a relatively serious setback as during the most intense storms the auroral oval and corresponding ionospheric fluctuations causing large GIC expand to latitudes well below the lower boundary mapped from $3 R_{E}$ (for an extreme case, see Tsurutani et al., 2003). Although, for computational reasons, it may not be feasible to push the inner boundary of the MHD much below $3 R_{E}$, this is the first obvious issue that needs to be addressed to enhance the usability of the global magnetospheric MHD for storm-time GIC modeling. This matter will be investigated in detail in the forthcoming studies that will include inner magnetospheric physics modules to the modeling chain.

In Fig. 4 we present the x-component (geographic north) of the measured and the modeled magnetic field fluctuations at the stations shown in Fig. 3. Note that the modeled fields are not only due to the external sources but via the process
Date: 2003/10/25, Time: 02:24 UT

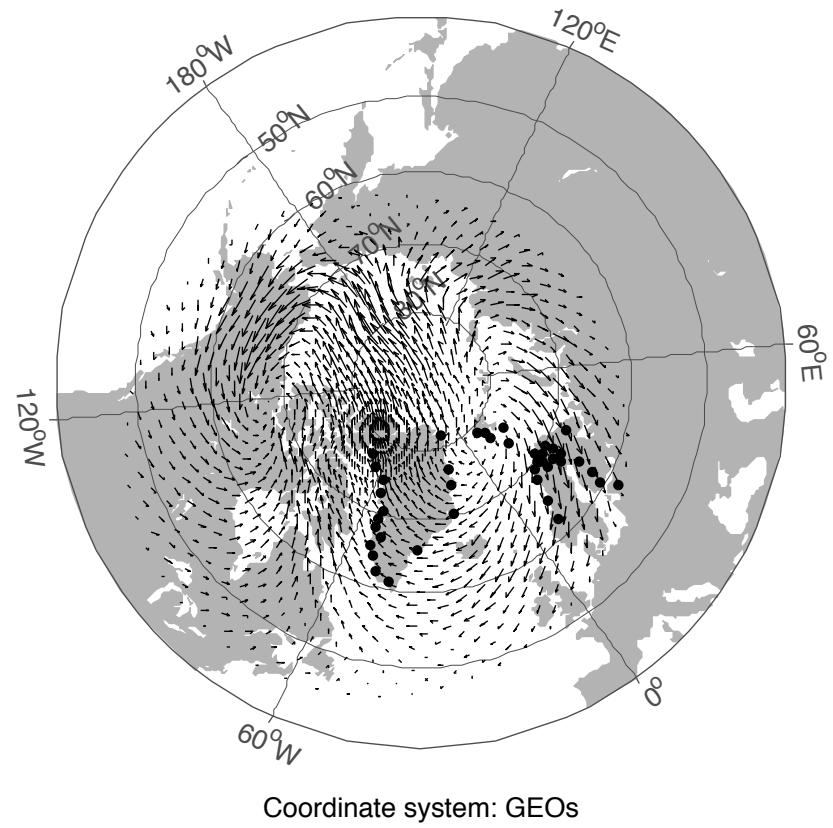

Fig. 3. Snapshot of the horizontal ionospheric currents given by the BATS-R-US. Dots indicate the locations to which the ground fields are computed and from which the measured ground magnetic field data was obtained. The date and the time of the snapshot is indicated in the title of the figure.

described in the previous section, also the internal contributions are included now. As a first-order approximation, the same conductivity model of central Finland (e.g., Viljanen et al., 1999) was used for all stations; other conductivity models can be added later.

The complex multiscale nature of auroral geomagnetic fluctuations is clearly seen in Fig. 4: wide variety of temporal and spatial scales are present in both the measured and the modeled data. The multiscale nature of the fluctuations makes a good visual presentation of the raw time series difficult as smaller scale features tend to smear the larger scale behavior. Thus, to better show the behavior at larger scales, we carried out moving average filtering (with moving 120 min windows) of the data in Fig. 4. The filtered data is shown in Fig. 5.

The first general observation from Figs. 4 and 5 is that although at the end of the event the model chain fails to reproduce the extreme amplitudes of the measured magnetic field fluctuations (maximum of $3673 \mathrm{nT}$ ), possibly associated with a substorm-type activity, the modeled field amplitudes seem to match to some extent with the measured field amplitudes. However, it is easily seen that the measured magnetic field appears to be more disturbed than the modeled field. Exceptions to this are the fluctuations at the beginning and at the end of the event where also the model produces very disturbed fields. By comparing the modeled magnetic 

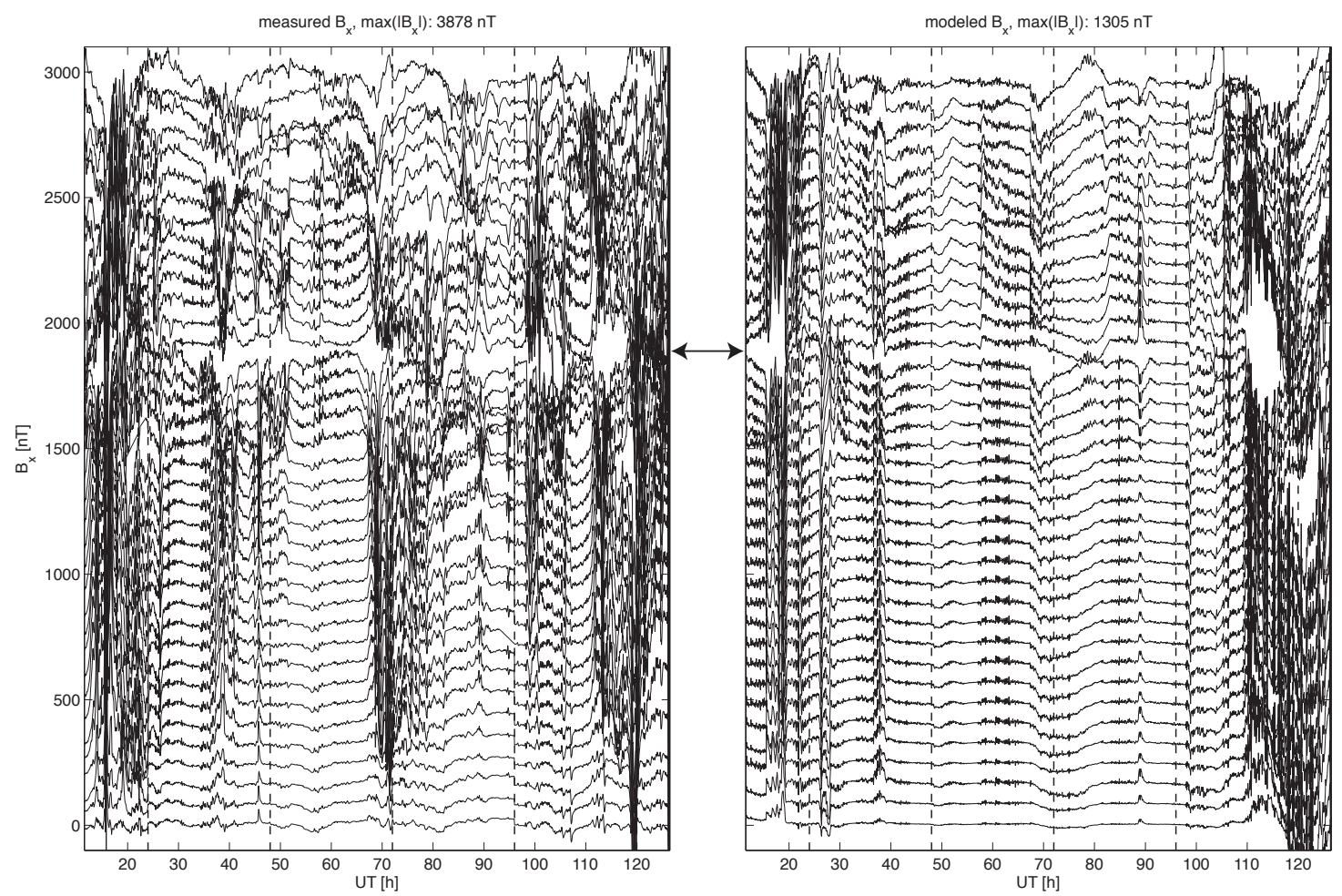

Fig. 4. The x-component (geographic north) of the measured (left panel) and the modeled (right panel) magnetic field fluctuations at stations shown in Fig. 3. Arrow in between the panels shows the separation between stations west of the Greenwich meridian (up from the arrow) and east of the Greenwich meridian (down from the arrow). Both sets are arranged from the geographically southernmost (bottom) to the northernmost (top) station. The time is UT hours from the beginning of 24 October 2003. The vertical dashed lines indicate the beginning of each new UT day.

fluctuations to the solar wind driver in Fig. 1, it is seen that the source for these, possibly global magnetospheric fluctuations is the highly turbulent IMF.

Another possible problem with the MHD-based modeling is underlined by the activity observed around hour 70 after 24 October, 00:00 UT seen in Figs. 4 and 5, which is to a large extent absent in the modeled time series. It should be noted that as at least part of the observed activity around hour 70 may be associated with substorm-type physical processes, which may be partially non-MHD, this particular activity may be out of the scope of global MHD modeling (more discussion about this below). Anyhow, despite the obvious differences, we conclude that the overall visual spatiotemporal morphology of the measured and the modeled magnetic field fluctuations seems to be quite similar.

We then move to take a look at the geomagnetically induced electric fields and currents. First, we model the "measured" electric field with the plane wave method (Cagniard, 1953) by using the measured ground magnetic field fluctuations and the ground conductivity model of central Finland. In the absence of steep lateral ground conductivity gradients, the electric field modeled this way corresponds relatively closely to the actual meso-scale $(\sim 100 \mathrm{~km})$ field (see e.g., Viljanen et al., 2004). In fact, the modeled electric field is often more representative of the meso-scale fields for GICrelated applications than the real measured electric field. The reason for this is that the electric field measurements using electrode distances of the order of hundreds of meters is often significantly distorted by the local inhomogeneities of the ground conductivity. Such distortions tend to smear the electric field of our interest and thus generally such measurements are of no use in GIC studies.

Once we have obtained both the modeled and the "measured" electric fields at the stations shown in Fig. 3, we insert them into the equation

$\mathrm{GIC}=a E_{x}+b E_{y}$

where $E_{x}$ and $E_{y}$ are the horizontal components of the electric field and $a$ and $b$ are constant coefficients. Equation (1) is used to model GIC fluctuations in technological systems when the local electric field is known. The coefficients $a$ and $b$ depend on the electrical, geometrical and topological properties of the system of interest and they can be deduced empirically and/or theoretically (Lehtinen and Pirjola, 1985; Pulkkinen et al., 2001a, 2006b). We will use coefficients $a=-70 \mathrm{Akm} / \mathrm{V}$ and $b=88 \mathrm{Akm} / \mathrm{V}$ that have been deduced 


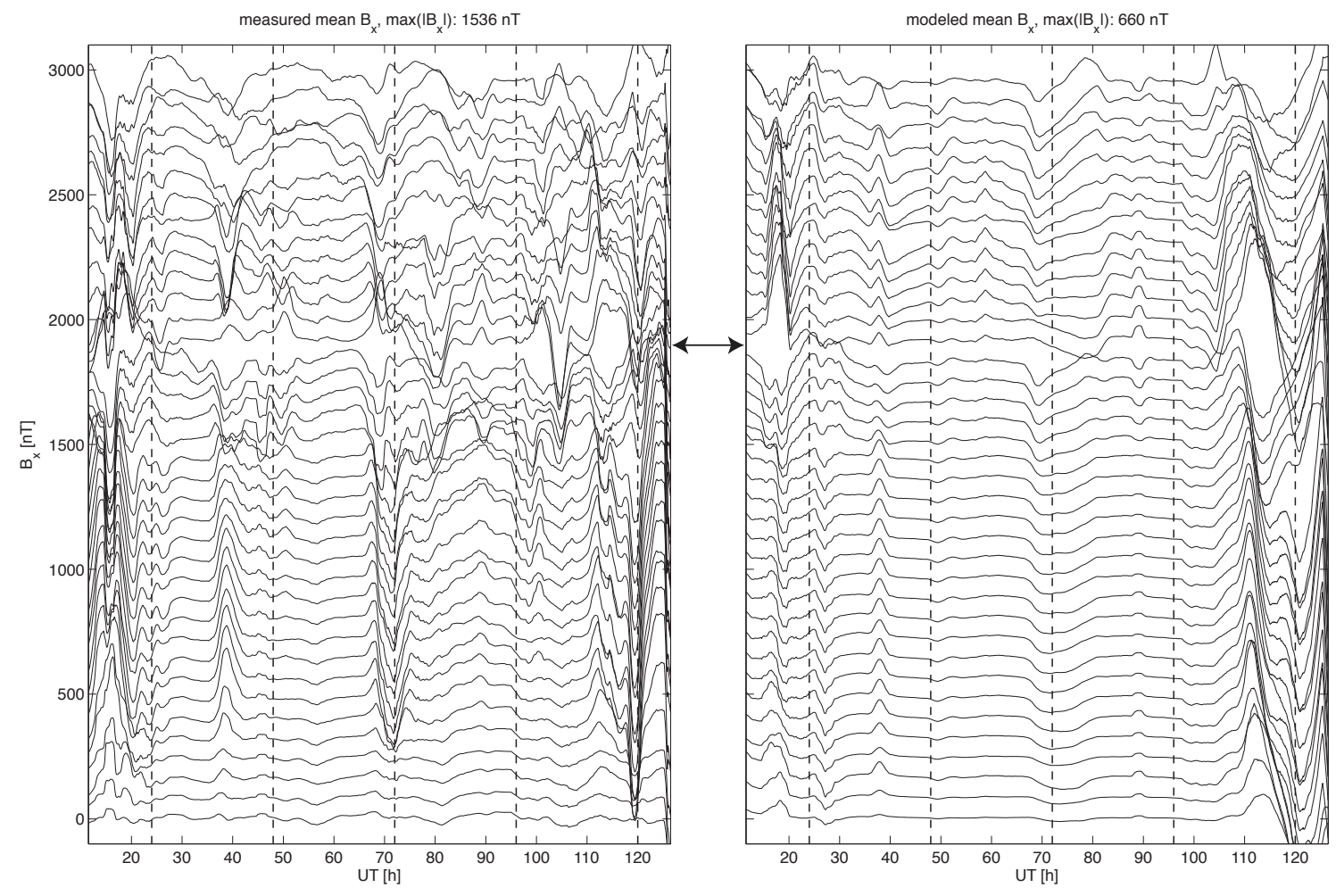

Fig. 5. Average magnetic field fluctuations for data shown in Fig. 4. The averaging was carried out in moving windows having length of $120 \mathrm{~min}$.

for the Mäntsälä section of the Finnish natural gas pipeline (Pulkkinen et al., 2001b). By using the same ground conductivity model and the same coefficients $a$ and $b$, we essentially assume that the ground and the network conditions are identical for all stations which is, of course, a crude approximation. However, as our primary interest at this point is in testing the capability of the MHD to produce realistic ionospheric current fluctuations driving the geomagnetic induction process, the approach can be considered acceptable.

At this point we re-emphasize that due to the 4-min temporal resolution of the MHD data, all data presented here are averaged to the 4-min resolution. As was shown by Pulkkinen et al. (2006b), temporal averaging of the magnetic field data beyond of about 1-min resolution may reduce the capability of the data to produce the highest GIC peaks; our rough estimation is that averaging to the 4-min resolution may reduce the peak values of the computed auroral GIC to about 50 per cent of their 10 -s values. Thus, in the future modeling efforts one should prefer the usage of higher temporal resolution MHD data.

In Fig. 6 we show the "measured' and the modeled GIC at all stations. It is again seen that the multiscale nature of the fluctuations makes good visual presentation of the raw data difficult. As the noise-like character of GIC makes computation of the $120 \mathrm{~min}$ averages quite meaningless, we characterize the fluctuations by computing moving standard de- viation (with moving $120 \mathrm{~min}$ windows) of the data instead. The results of these computations are shown in Fig. 7.

The basic observations from Figs. 6 and 7 are similar to those from Figs. 4 and 5: although the peak values are smaller, the modeled GIC can obtain amplitudes comparable to those of the "measured" GIC and the basic spatiotemporal morphology of GIC is quite similar. Taking into account the more disturbed measured field in Fig. 4 and the fact that the time derivative of the magnetic field is a quite good proxy for the GIC activity (Viljanen et al., 2001), the morphological correspondence between the GIC can be considered, in the context of complex auroral ionospheric phenomena, quite good. From the qualitative viewpoint, we may conclude that for this particular event and with this particular model setup, the global magnetospheric MHD seems to be able to reproduce some of the basic features of GIC.

\section{Utility metrics-based quantitative validation of the model performance}

Next we want to transfer the qualitative view of Figs. 4-7 into a quantitative one. Here we need to put some special consideration for appropriate metrics. We note that this part of the study builds on the work by Weigel et al. (2006) (and 

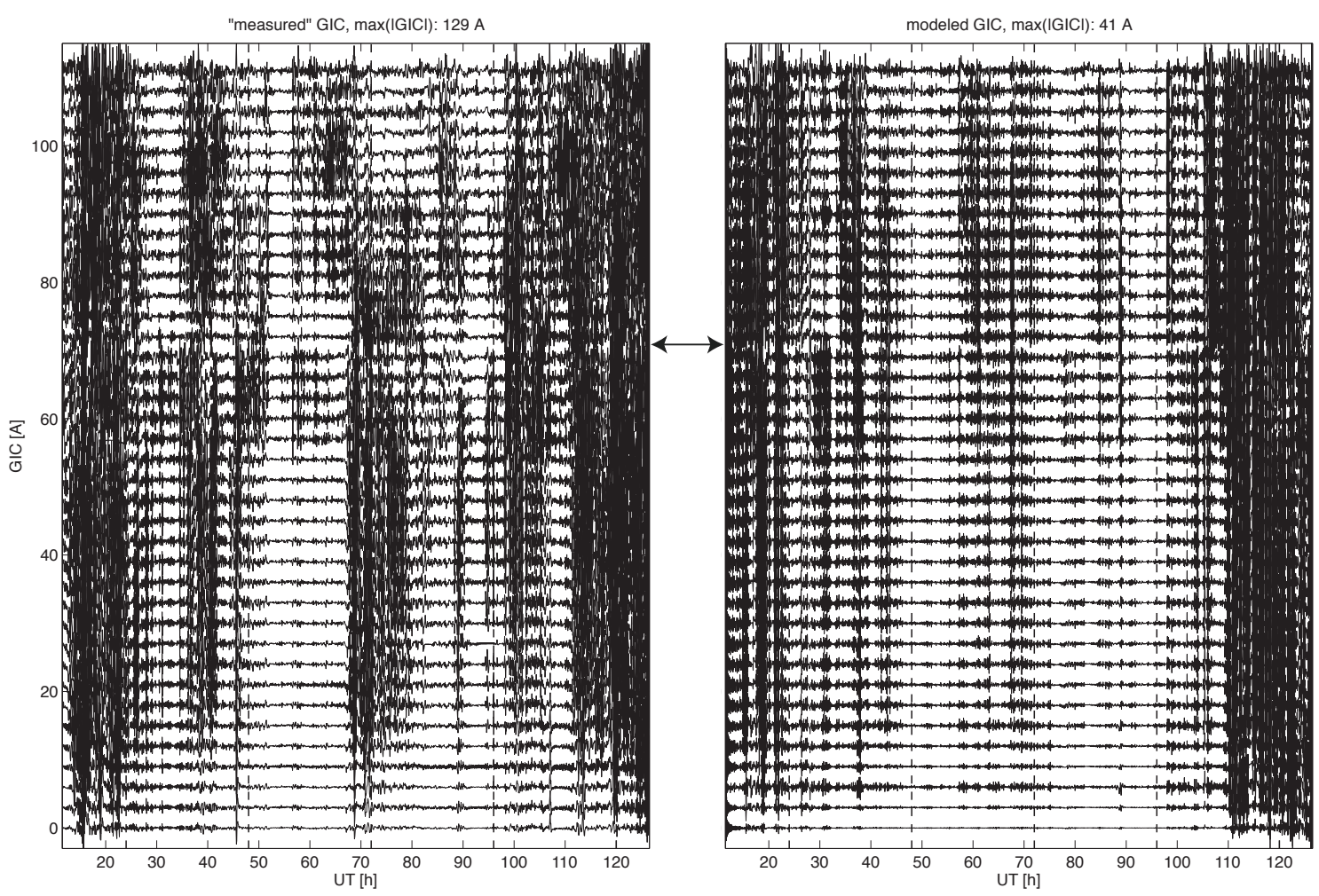

Fig. 6. "Measured" (see the text for explanation) and the modeled GIC at stations shown in Fig. 3. Arrow in between the panels shows the separation between stations west of the Greenwich meridian (up from the arrow) and east of the Greenwich meridian (down from the arrow). Both sets are arranged from the geographically southernmost (bottom) to the northernmost (top) station. The time is UT hours from the beginning of 24 October 2003. The vertical dashed lines indicate the beginning of each new UT day.

references therein) and the reader is referred there for more details on the discussed metrics.

In physics, by far the most popular metrics are correlationbased, like that of the mean squared difference. Such metrics are particularly suitable for, for example, model optimization due to their convenient mathematical properties. However, in our case these basic metrics are not ideal for two different reasons: 1.) due to the complexity of the GIC signal and 2.) due to the lack of user-relevance. As can be qualitatively seen from Fig. 6 and as has been quantitatively verified for the time derivative of the ground magnetic field fluctuations by Pulkkinen et al. (2006a), the GIC signal is very complex. In fact, some of the statistical properties of the signal resemble that of the white noise. It follows, that for such highly fluctuating signal, for example, linear correlation may not be a "fair" metric for evaluating the model performance. Thus, a metric focusing on some more overall aspect of the signal, like mere amplitude, should be preferred. On the other hand, from the viewpoint of the user, one should prefer metrics that has something to do with the actual decision process involving the forecast $\rightarrow$ mitigation action.

Quite conveniently, both of the aforementioned issues are addressed by the so-called utility metrics. The utility of the forecast is defined as

$U_{f}=B N_{H}-C N_{\bar{H}}$

where $N_{H}$ is the number of correct forecasts, $N_{\bar{H}}$ is the number of false alarms, $C$ is the cost of taking mitigating action and $B$ is the benefit from having taken mitigating action when an event occurred. In using this particular metric we assume that 1.) the user takes the same mitigating action following each forecast of an event, 2.) an "always mitigate" strategy yields a net monetary loss for the user and 3.) the user seeks to maximize the monetary gain $U_{f}$. Note that sometimes it may be preferable for the user to change the system in a way that removes the threat altogether thus violating the assumption no. 2. Although such actions can be carried out to mitigate GIC (Molinski, 2002), often the costs of changing the system cannot be justified by the size of the threat posed by GIC. Then the assumption no. 2 holds and it may be beneficial for the user to seek for monetary gain $U_{f}$ in terms of GIC forecasts. Also note that we are considering utility with respect to a system that is never mitigated, which is the case for most of the systems experiencing GIC. In another words, we are considering the difference between losses/gains experienced by the two systems. It follows that we do not need to consider missed events; a missed event will 

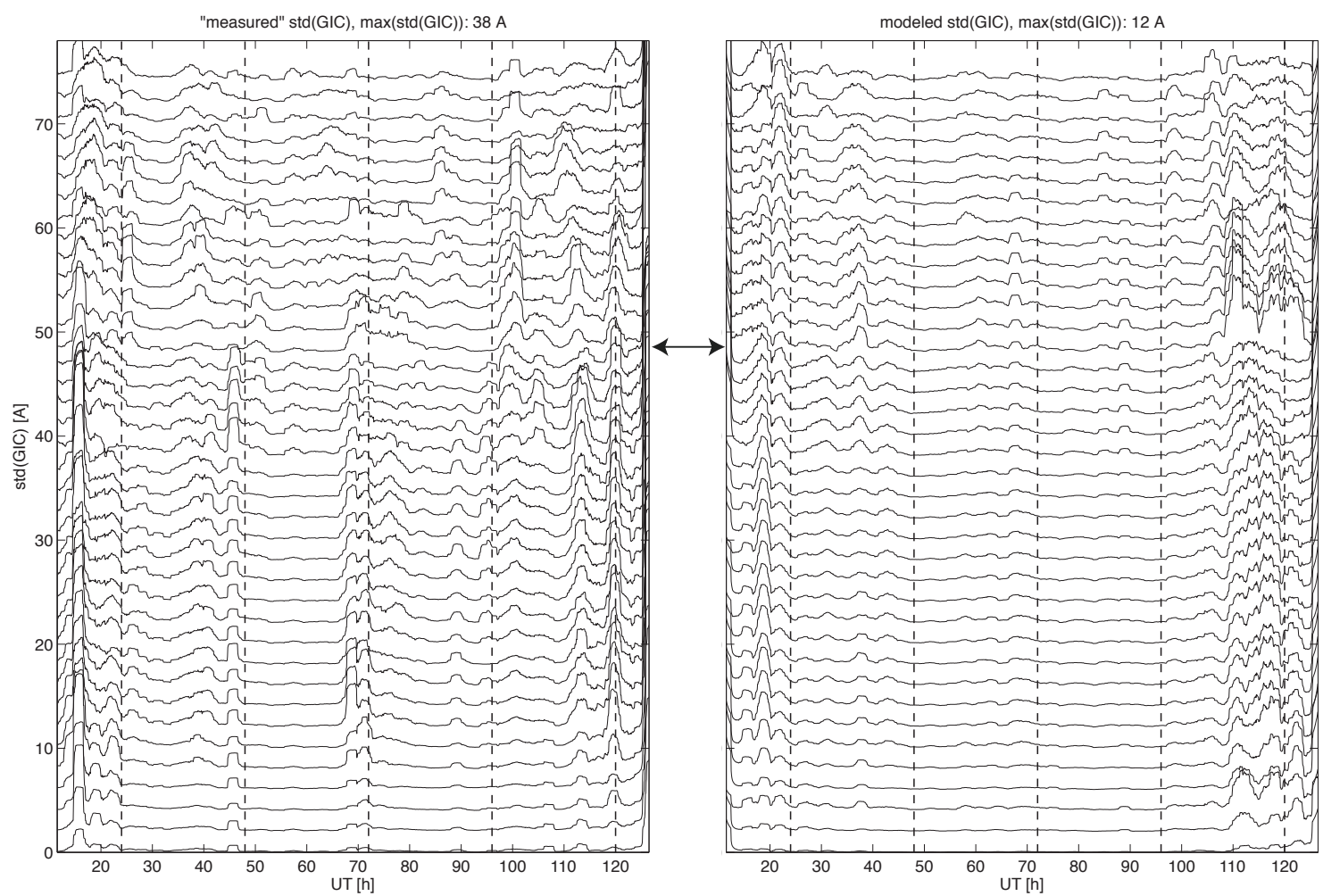

Fig. 7. Standard deviation of GIC data in Fig. 6. The standard deviation was computed for moving windows having length of 120 min.

cause the same monetary loss for both the reference system and the system using mitigation actions.

In using the utility, we evaluate the model performance in terms of its capability to predict "events". Here we define an event as follows: within a forecast window $0 \leq t \leq t_{f}$, the absolute value of GIC exceeds event threshold $\left|\mathrm{GIC}_{\text {thres }}\right|$ (see Fig. 8). The windows are moved over the time series in nonoverlapping parts and events for given $t_{f}$ and $\left|\mathrm{GIC}_{\text {thres }}\right|$ are recorded for both the measured and the modeled GIC. As values for $B$ and $C$ in Eq. (2) are system dependent and are estimated by the user, rather than computing $U_{f}$, we compute the forecast ratio $R_{f}=N_{H} / N_{\bar{H}}$. It is easily seen that the utility $U_{f}$ is positive if $R_{f}>C / B$ and thus by reporting $R_{f}$ of the forecast the user can quantify the utility of the forecast once the values $B$ and $C$ and are known. In model comparisons, a model with a larger $R_{f}$ will have a greater utility $U_{f}$.

In Fig. 9 we show $R_{f}$ for the modeled GIC and for two different persistence models/rules as a function of $\left|\mathrm{GIC}_{\text {thres }}\right|$ for $t_{f}=32 \mathrm{~min}$ and $t_{f}=60 \mathrm{~min}$. The persistence models are: 1.) "always an event forecast", i.e. during disturbed periods the alarm is always on. Note that this is not necessarily the same as taking mitigation action always; during quiet conditions the system may not be at the state of GIC mitigation. The alarm is switched on, for example, only when an approaching interplanetary coronal mass ejection is observed.
2.) "nearest neighbor forecast", i.e. the forecast for the next forecast window will be the same as the observation for the previous one. $R_{f}$ were computed separately for each station, we show here only the average taken over all of the stations. It is noted that there are differences in $R_{f}$ between different stations especially as a function of the latitude. However, the basic trend is the same for all stations and thus the average $R_{f}$ gives a quite good idea about the overall model performance.

As seen from Fig. 9, the forecast ratios behave similarly for both 32 - and 60-min forecast windows. The only clear difference is that the utility tends to be slightly higher for the 60-min forecasts with smaller event threshold $\left(\left|\mathrm{GIC}_{\text {thres }}\right|<5 \mathrm{~A}\right)$. Interestingly, for event thresholds $\left|\mathrm{GIC}_{\text {thres }}\right|>5.5 \mathrm{~A}$, the MHD-based forecasts have the greatest utility for 32-min forecasts. In general, it is seen that the firstprinciples modeling process is capable of producing equal, and in some cases even superior economic benefit to that of the used persistence models. Considering the potential for improving the modeling accuracy by adding new model components and by using higher spatial resolution in the global magnetospheric MHD, this result is promising. Whether or not the potential can be realized, will be investigated in the continuation of this work. 


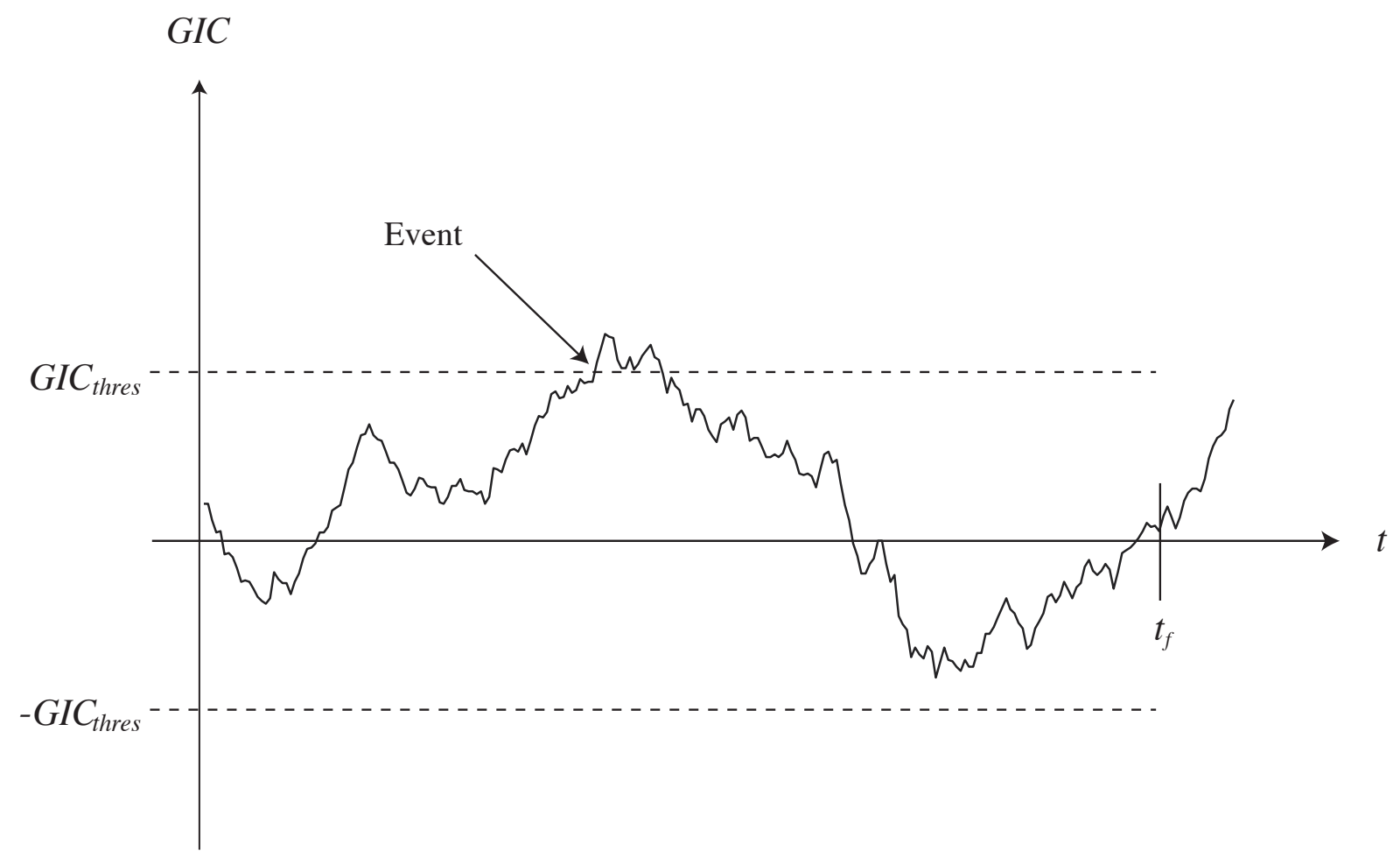

Fig. 8. The determination of an "event" from the GIC time series. The event is defined as a crossing of the event threshold $\mid$ GIC thres $\mid$ within a forecast window $0 \leq t \leq t_{f}$.

\section{Summary and discussion}

Empirical modeling of the ground magnetic field fluctuations, which via Faraday's law of induction are closely related to the geomagnetically induced electromagnetic fields and currents, has long traditions in the modern space physics (e.g., Bargatze et al., 1985; Vassiliadis et al., 1995; Valdivia et al., 1999; Weimer, 2005). These efforts have not been in vain; there has been great success in predicting some overall aspects of geomagnetic field fluctuations, like those of the global geomagnetic indices, and the models have been able to give crucial insights into the physical characteristics of the solar wind-magnetosphere-ionosphere system. However, accurate prediction of the geomagnetic induction-related field fluctuations requires going beyond the overall aspects. More specifically, one needs to know the rate of the change of the ground magnetic field at the temporal scales of the order of minutes; as the variations around the mean are usually more complex than the mean behavior itself, it is thus quite clear that extracting such information poses a new challenge for the geomagnetic field modeling. Importantly, some recent empirical modeling efforts have had some success in predicting the rapidly fluctuating part of the geomagnetic field (Weigel et al., 2003; Wintoft, 2005). These models naturally have potential for usage in space weather forecasting applications.
As the empirical models have been "taught" to reproduce the measurements, it is clear that in some situations the empirical models outperform the first-principles models. However, in situations that the empirical models have not "seen", they may extrapolate very poorly whereas first-principles models always, in principle, produce physically reasonable results provided that the basic physics of the system remain unchanged. This is especially important in rare and extreme situations that naturally in the context of space weather are of the utmost importance (see also, Lopez et al., 2007). From the physics viewpoint, the performance of our first-principles models is the ultimate test for our understanding of the solar wind-magnetosphere-ionosphere system; the system's evolution equations are derived using our understanding of the underlaying elementary physical principles rather than by the data.

The point of the above discussion is, while acknowledging the great significance of the empirical modeling efforts, to emphasize the importance of pursuing towards firstprinciples-based modeling of the solar wind-magnetosphereionosphere system. Accordingly, the goal of this work was to carry out first-principles-based modeling of near-space phenomena from the upstream solar wind to the surface of the Earth. More specifically, we introduced a basic modeling procedure for obtaining the geomagnetically induced electromagnetic fields and currents from the output of global 
magnetospheric MHD codes. We also presented metrics that take into account both the complex nature of the signal and possible forecasting applications of the modeling process. The modeling process and the metrics were introduced with the help of an actual example space weather event of 24 29 October 2003. Observations of the ground magnetic field fluctuations were used to carry out preliminary tests of the performance of the model chain. As space weather applications are the main motivation of the study, the performance tests were focused on GIC.

It was seen that for the studied event, some central features of the overall ionospheric current fluctuations associated with GIC were captured by the modeling process. More specifically, the basic spatiotemporal morphology of the modeled and "measured" GIC was quite similar. Furthermore, the presented user-relevant utility metrics demonstrated that global MHD-based modeling can outperform simple GIC persistence models. We may thus conclude that considering the relatively modest model setup, the first results are encouraging.

It should be noted, however, that despite the success in reproducing some of the observed features of the GIC-related ionospheric current fluctuations, there were also important shortcomings. First, the $3 R_{E}$ inner magnetospheric boundary of the global MHD run does not extend very low in magnetic latitude in the ionosphere. This is a setback as during strong storms, in addition to generally very disturbed polar regions, the auroral oval with highly fluctuating ionospheric currents generating large GIC may expand below the ionospheric MHD boundary. However, this problem may be scaled back by pushing the inner boundary of the global MHD closer to the Earth and by including inner magnetospheric model to the modeling chain. Inclusion of the inner magnetosphere may be important also in terms of having more realistic dynamics associated with the region 2 current system (e.g., De Zeeuw et al., 2004).

It is emphasized that realistic modeling of the ionospheric currents is perhaps the most important factor influencing the accuracy of the computed induced fields. Thus, it is obvious that inclusion of as many as possible central physical elements contributing to the magnetosphere-ionosphere coupling is highly desirable. However, it is clear that some physics may be missing partially or altogether in the MHDbased description of the system; also this was demonstrated in the example event. For example, it is well-known that some processes in the plasma sheet are beyond the scope of MHD. Furthermore, these processes may play a crucial role, for example, in the substorm phenomenon which is known to be statistically one of the most important causes for large high-latitude GIC (e.g., Viljanen et al, 2006a). Although there is evidence that global MHD can produce substorm-like behavior of the magnetosphere (e.g., Raeder et al., 2001a; Wiltberger et al., 2005), it is all but clear if the highly complex nature of the ground magnetic field variations associated with substorms (for a quantification of this complexity, see
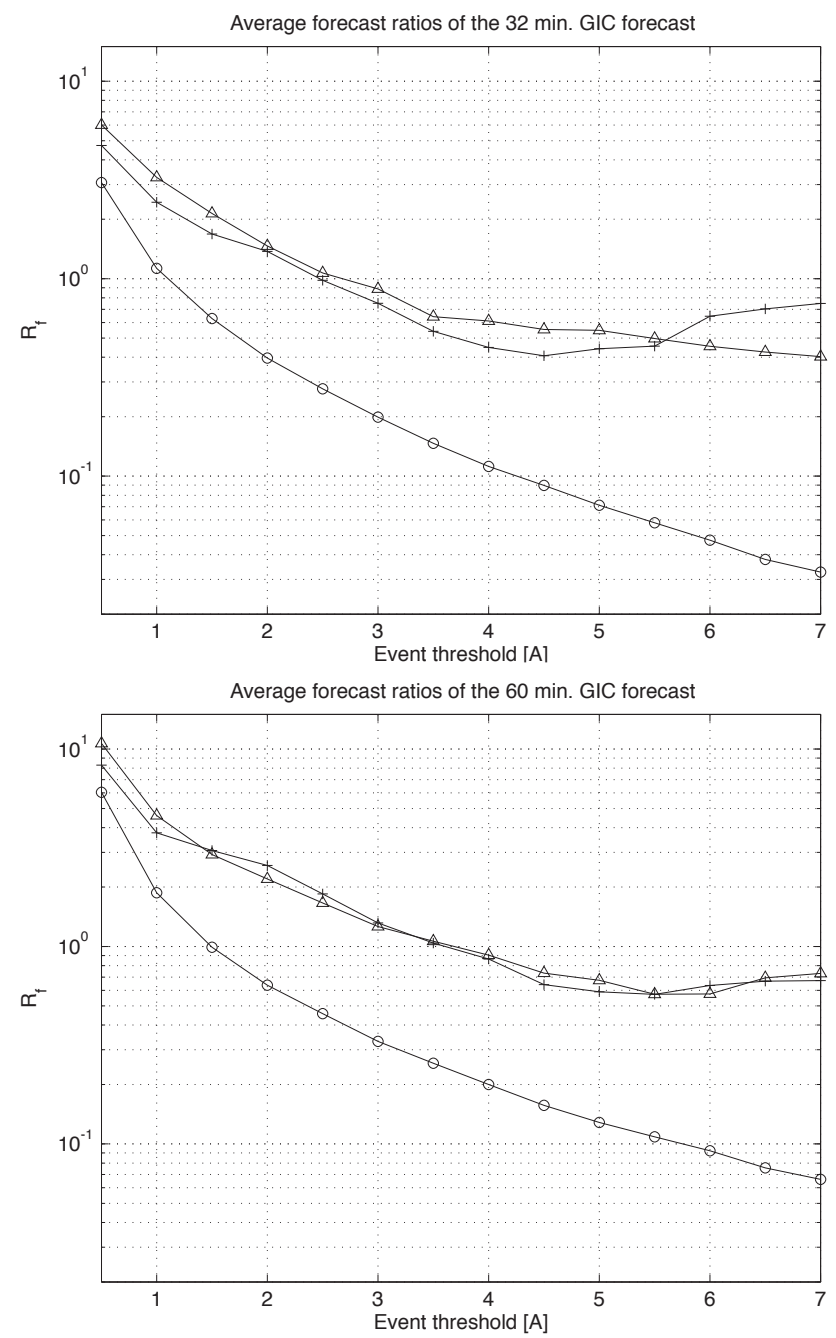

Fig. 9. Forecast ratios $N_{H} / N_{\bar{H}}$ of the modeled GIC (plusses) and persistence models 1 (circles) and 2 (triangles) as a function of the event threshold $\left|\mathrm{GIC}_{\text {thres }}\right|$. Top panel: forecast window $t_{f}$ of $32 \mathrm{~min}$ was used. Bottom panel: forecast window $t_{f}$ of $60 \mathrm{~min}$ was used.

Pulkkinen et al., 2006a) can be reproduced by basic global magnetospheric MHD models (for an alternative approach, see e.g., Klimas et al., 2004). Obviously, capturing these extreme variations is very important from the GIC viewpoint.

As mentioned above, the work at hand comprises Phase 1 of the project in which we test the present capabilities of first-principles modeling of space weather from the ground viewpoint. In the follow-up, we will utilize numerous setups and combinations of the models hosted by the Community Coordinated Modeling Center (CCMC) operating at NASA Goddard Space Flight Center to find the optimal way to carry out the modeling. The optimization will be carried out by applying user-relevant metrics, like that of the presented utility metrics. 
Acknowledgements. A. Viljanen and J. Waterman of Finnish Meteorological Institute and Danish Meteorological Institute, respectively, are acknowledged for providing the IMAGE and Greenland magnetometer array data utilized in this study. Comments on the manuscript by A. Viljanen and R. Pirjola are greatly acknowledged. CIM software by A. Viljanen was used in this work.

Topical Editor M. Pinnock thanks J. Watermann and S. G. Shepherd for their help in evaluating this paper.

\section{References}

Avdeev, D. B., Kuvshinov, A. V., Pankratov, O. V., and Newman, O. G.: Three-dimensional induction logging problems, Part I: An integral equation solution and model comparisons, Geophysics, 67, 2, 413-426, 2002.

Bargatze, L. F., Baker, D. N., McPherron, R. L., and Hones, E. W.: Magnetospheric impulse response for many levels of geomagnetic activity, J. Geophys. Res., 90(A7), 6387-6394, 1985.

Bargatze, L. F., McPherron, R. L., Minamora, J., and Weimer, D.: A new interpretation of Weimer et al.'s solar wind propagation delay technique, J. Geophys. Res., 110, A07105, doi:10.1029/2004JA010902, 2005.

Beamish, D., Clark, T. D. G., Clarke, E., and Thomson, A. W. P.: Geomagnetically induced currents in the UK: geomagnetic variations and surface electric fields, J. Atmos. Sol. Terr. Phys., 64, 1779-1792, 2002.

Bolduc, L.: GIC observations and studies in the Hydro-Qubec power system, J. Atmos. Solar-Terr. Phys., 64, 16, 1793-1802, 2002.

Boteler, D. H. and Pirjola, R. J.: The complex-image method for calculating the magnetic and electric fields produced at the surface of the Earth by the auroral electrojet, Geophys. J. Int., 132, 1, 31-40, 1998.

Boteler, D. H., Pirjola, R. J., and Nevanlinna, H.: The Effects of Geomagnetic Disturbances on Electrical Systems at the Earth's Surface, Adv. Space Res., 22, 17-27, 1998.

Boteler, D. H., Trichtchenko, L., Pirjola, R., Parmelee, J., Souksaly, S., Foss, A., and Marti, L.: Real-time simulation of geomagnetically induced currents, Proceedings of the Canadian Conference on Electrical and Computer Engineering, IEEE Canada, Ottawa, Ontario, Canada, 7-10 May 2006, 4 pp, 2006.

Cagniard, L.: Basic theory of the magneto-telluric method of geophysical prospecting, Geophysics, 18(3), 605-635, 1953.

Czech, P., Chano, S., Huynh, H., and Dutil, A.: The Hydro-Québec System Blackout of 13 March 1989: System Response to Geomagnetic Disturbance, EPRI Report, TR-100450, Proceedings of Geomagnetically Induced Currents Conference, Millbrae, California, USA, 8-10 November 1989, 1992.

De Zeeuw, D. L., Sazykin, S., Wolf, R. A., Gombosi, T. I., Ridley, A. J., and Tóth, G.: Coupling of a global MHD code and an inner magnetospheric model: Initial results, J. Geophys. Res., 109, A12219, doi:10.1029/2003JA010366, 2004.

Dmitriev, A. V., Chao, J.-K., Suvarova, A. V., Ackerson, K., Ishisaka, K., Kasaba, Y., Kojima, H., and Matsumoto, H.: Indirect estimation of the solar wind conditions in 29-31 October 2003, J. Geophys. Res., 110, A09S02, doi:10.1029/2004JA010806, 2005.

Klimas, A. J., Uritsky, V. M., Vassiliadis, D., and Baker, D. N.: Reconnection and scale-free avalanching in a driven current-sheet model, J. Geophys. Res., 109, A02218, doi:10.1029/2003JA010036, 2004.

Lehtinen, M. and Pirjola, R.: Currents produced in earthed conductor networks by geomagnetically-induced electric fields, Ann. Geophys., 3(4), 479-484, 1985.

Lopez R. E., Hernandez, S., Wiltberger, M., Huang, C.-L., Kepko, E. L., Spence, H., Goodrich, C. C., and Lyon, J. G.: Predicting magnetopause crossings at geosynchronous orbit during the Halloween storms, Space Weather, 5, S01005, doi:10.1029/2006SW000222, 2007.

Molinski, T.: Why utilities respect geomagnetically induced currents, J. Atmos. Sol-Terr. Phys., 64, 1765-1778, 2002.

Olsen, N. and Kuvshinov, A.: Modeling the ocean effect of geomagnetic storms, Earth Planets Space, 56, 525-530, 2004.

Pirjola, R. and Viljanen, A.: Complex image method for calculating electric and magnetic fields produced by and auroral electrojet if finite length, Ann. Geophys., 16, 1434-1444, 1998, http://www.ann-geophys.net/16/1434/1998/.

Pirjola, R., Viljanen, A., Pulkkinen, A., Kilpua, S., and Amm, O.: Space weather effects on electric power transmission grids and pipelines, NATO Science Series, Volume "Effects of Space Weather on Technology Infrastructure", Kluwer Academic Publishers B.V., edited by: Daglis, I., Chapter 13, 235-256, 2004.

Powell, K. G., Roe, P. L., Linde, T. J., Gombosi, T. I., and De Zeeuw, D. L.: A solution-adaptive upwind scheme for ideal magnetohydrodynamics, J. Comput. Phys., 154(2), 284, doi:10.1006/jcph.1999.6299, 1999.

Pulkkinen, A., Pirjola, R., Boteler, D., Viljanen, A., and Yegorov, I.: Modelling of space weather effects on pipelines, J. Appl. Geophys., 48, 233-256, 2001a.

Pulkkinen, A., Viljanen, A., Pajunpää, K., and Pirjola, R.: Recordings and occurrence of geomagnetically induced currents in the Finnish natural gas pipeline network, J. Appl. Geophys., 48, 219-231, 2001b.

Pulkkinen, A. and Engels, M.: The role of 3D geomagnetic induction in the determination of the ionospheric currents from the ground geomagnetic data, Ann. Geophys., 23, 909-917, 2005, http://www.ann-geophys.net/23/909/2005/.

Pulkkinen, A., Lindahl, S, Viljanen, A., and Pirjola, R.: Geomagnetic storm of 2931 October 2003: Geomagnetically induced currents and their relation to problems in the Swedish high-voltage power transmission system, AGU Space Weather, 3, S08C03, doi:10.1029/2004SW000123, 2005.

Pulkkinen, A., Klimas, A., Vassiliadis, D., Uritsky, V., and Tanskanen, E.: Spatiotemporal scaling properties of the ground geomagnetic field variations, J. Geophys. Res., 111, A03305, doi:10.1029/2005JA011294, 2006a.

Pulkkinen, A., Viljanen, A., and Pirjola, R.: Estimation of geomagnetically induced current levels from different input data, Space Weather, Vol. 4, S08005, doi:10.1029/2006SW000229, $2006 \mathrm{~b}$.

Raeder, J., McPherron, R. L., Frank, L. A., Kokubun, S, Lu, G., Mukai, T., Paterson, W. R., Sigwarth, J. B., Singer, H. J., and Slavin, J. A.: Global simulation of the Geospace Environment Modeling substorm challenge event, J. Geophys. Res., 106(A1), 381-396, doi:10.1029/2000JA000605, 2001a.

Raeder, J., Wang, Y., Fuller-Rowell, T., and Singer, H. J.: Global simulation of space weather effects of the Bastille Day storm, Solar Physics, 204, 325, 2001 b.

Ridley, A. J., Gombosi, T. I., and De Zeeuw, D. L.: Ionospheric 
control of the magnetospheric configuration: Conductance, Ann. Geophys., 22, 567-584, 2004,

http://www.ann-geophys.net/22/567/2004/.

Skoug R. M., Gosling, J. T., Steinberg, J. T., McComas, D. J., Smith, C. W., Ness, N. F., Hu, Q., and Burlaga, L. F.: Extremely high speed solar wind: 29-30 October 2003, J. Geophys. Res., 109, A09102, doi:10.1029/2004JA010494, 2004.

Tóth, G., Sokolov, I., Gombosi, T., Chesney, D., Clauer, C.R., De Zeeuw, D., Hansen, K., Kane, K., Manchester, W., Oehmke, R., Powell, K., Ridley, A., Roussev, I., Stout, Q., Volberg, O., Wolf, R., Sazykin, S., Chan, A., Yu, B., and Kóta, J.: Space Weather Modeling Framework: A new tool for the space science community, J. Geophys. Res., 110, A12226, doi:10.1029/2005JA011126, 2005,

Tsurutani B. T., Gonzalez, W. D., Lakhina, G. S., and Alex, S.: The extreme magnetic storm of 12 September 1859, J. Geophys. Res., 108(A7), 1268, doi:10.1029/2002JA009504, 2003.

Untiedt, J. and Baumjohann, W.: Studies of polar current systems using the IMS Scandinavian magnetometer array, Space Sci. Rev., 63, 245-390, 1993.

Valdivia, J. A., Vassiliadis, D., Klimas, A., Sharma, A. S., and Papadopoulos, K.: Spatiotemporal activity of magnetic storms, J. Geophys. Res., 104(A6), 12 239-12 250, 10.1029/1999JA900152, 1999.

Vassiliadis, D., Klimas, A. J., Baker, D. N., and Roberts, D. A.: A description of the solar wind-magnetosphere coupling based on nonlinear filters, J. Geophys. Res., 100(A3), 3495-3512, doi:10.1029/94JA02725, 1995.

Weigel, R. S., Klimas, A. J., and Vassiliadis, D.: Solar wind coupling to and predictability of ground magnetic fields and their time derivatives, J. Geophys. Res., 108(A7), 1298, doi:10.1029/2002JA009627, 2003.
Weigel, R. S., Detman, T., Rigler, E. J., and Baker, D. N.: Decision theory and the analysis of rare event space weather forecasts, Space Weather, 4, S05002, doi:10.1029/2005SW000157, 2006.

Weimer, D. R.: Predicting surface geomagnetic variations using ionospheric electrodynamic models, J. Geophys. Res., 110, A12307, doi:10.1029/2005JA011270, 2005.

Wiltberger, M., Elkington S. R., Guild, T., Baker, D. N., and Lyon, J. G.: Comparison of MHD simulations of isolated and storm time substorms, AGU Geophysical Monograph Series, Inner Magnetosphere: Physics and Modeling, 155, 271-281, 2005.

Wintoft, P.: Study of solar wind coupling to the time difference horizontal geomagnetic field, Ann. Geophys., 23, 1949-1957, 2005, http://www.ann-geophys.net/23/1949/2005/.

Viljanen, A., Pirjola, R., and Amm, O.: Magnetotelluric source effect due to $3 \mathrm{D}$ ionospheric current systems using the complex image method for 1D conductivity structures, Earth, Planets and Space, 51, 933-945, 1999.

Viljanen, A., Nevanlinna, H., Pajunpää, K., and Pulkkinen, A.: Time derivative of the horizontal geomagnetic field as an activity indicator, Ann. Geophys., 19, 1107-1118, 2001, http://www.ann-geophys.net/19/1107/2001/.

Viljanen, A., Pulkkinen, A., Amm, O., Pirjola, R., Korja, T., and BEAR Working Group: Fast computation of the geoelectric field using the method of elementary current systems, Ann. Geophys. 22, 101-113, 2004, http://www.ann-geophys.net/22/101/2004/.

Viljanen, A., Tanskanen, E., and Pulkkinen, A.: Relation between substorm characteristics and rapid temporal variations of the ground magnetic field, Ann. Geophys., 24, 725-733, 2006a.

Viljanen, A., Pulkkinen, A., Pirjola, R., Pajunpää, K., Posio, P., and Koistinen, A.: Recordings of geomagnetically induced currents and a nowcasting service of the Finnish natural gas pipeline system, Space Weather, 4, S10004, doi:10.1029/2006SW000234, $2006 b$. 